\title{
RESERCH PROTOCOL
}

\section{ASSESSMENT OF COGNITIVE FUNCTIONS AND DEVELOPMENT OF COGNITIVE- BASED SMARTPHONE APPLICATION FOR MALAY PARKINSON DISEASE PATIENTS}

\author{
Wael MY Mohamed \\ Department of Basic Medical Sciences, Kulliyyah of Medicine, International Islamic University \\ Malaysia (IIUM), Kuantan, Malaysia \\ Clinical Pharmacology Department, Menoufia Medical School, Menoufia University, Menoufia, \\ Egypt
}

\section{Correspondence:}

Wael M. Y. Mohamed

wmy107@gmail.com

\section{Background:}

The number of elderly people worldwide is rising at an alarming rate, with almost 10,000 people turning 65 in the United States every day (Olshansky et al., 2009). This dramatic rise in the number of elderly people is also followed by an ever-increasing need for healthcare in the geriatric community (Olshansky et al., 2009).

The life span of the worldwide population had been increasing due to better health care system, diagnosis and increased awareness. Parkinson's disease (PD) is one of the most common age related neurodegenerative diseases (Hindle 2010).Neuronal loss both in genetic and sporadic form of PD is one of the major causes of PD and other neurodegenerative diseases. The biomarkers for the diagnosis and prognosis of PD are still not well defined hence the late diagnosis with reversal of the disease is difficult (Miller et. al. 2015). It is important to find novel mechanisms for prognosis and diagnosis as well as pathogenesis. The mechanisms of neuronal loss is still not clear in PD and needs to be investigated further to find out novel targets for therapeutic interventions.

To facilitate the clinical diagnosis of PD, numerous studies have focused on biomarkers based on neuroimaging and molecules in cerebrospinal fluid (CSF) and peripheral blood. For example, neuroimaging abnormalities detection by SPECT, meta-iodobenzyl guanidine I 123, sonography, PET, or MRI, have been tested in patients with moderate or advanced disease. These allow noninvasive tracking of molecular targets of relevance to neurodegeneration but the cost is too high to use these techniques as screening tools in the general population. Likewise, several blood and cerebrospinal fluid (CSF) biomarkers such as $\alpha$-synuclein and DJ-1 have been tested for the diagnosis of PD, but the outcomes have been inconsistent, possibly due to the highly heterogeneous study population, sample contamination, and non-standardized sampling processes.

In order to effectively address the health of the elderly, especially those with PD needs in the years to come, it will be important to establish strategies to track the health status of the elderly and to identify the most productive methods for remote surveillance, disease prevention and health care. Recent technological developments, such as television and telemedicine, have been especially useful in a variety of environments (Levine and Gorman, 1999; Rosser et al., 2001; Rajan, 2012; Clifford and Clifton, 2012; Rogante et al., 2010). 
The systems allow remote interactions between patients, physicians and students that are critical to the next generation of clinical research and therapy (Panayides et al., 2010; Koch, 2006). Smart phones are cell phones specialising in the production of a new generation of clinical research with additional computing capabilities/services (Fortney et al., 2011; Boulos et al., 2011).

In addition, the number of mobile and pervasive medical applications on smartphones in the general population is rising and can be increased to include clinical research. Several papers have recently defined potential for mobile apps in circumstances, control of prescription drugs, food intakes, depression, strokes, diabetes, rehabilitation, opioid enforcement and patient care (Doherty and Oh, 2012; Dufau et al., 2011; Gill et al., 2012; Martin et al., 2012; Mellone et al., 2012; Mellone te al., 2012; Klasnja and pratt, 2012; Worringham et al., 2011).

Moreover, amid numerous advancements in cell phone science, the utility and value of smartphone apps used for clinical research or care for the elderly remains almost unknown. The latest proposal focuses on the creation of a mobile app for PD patient memory, mobility and fragility. The purpose of this proposal is to create a new cognitive test (Touch Screen Technology Processing Speed) that is suitable for use in elderly people with remote testing capabilities. The significance of this proposal is the development of smartphone-based evaluation batteries for cognitive and motor function in patients with PD.

\section{Knowledge Gap and Clinical Implications:}

Degenerative disorders of the central nervous system (CNS), such as Parkinson's disease (PD), affect the human motor system and present a variety of deficits, such as cognitive impairment and motor dysfunction (Mack and Marsh, 2017). Examples of such dysfunctions include reduced speech (Ludlow et al., 1987), higher regular calorie consumption (Marder et al., 2009), increased rigidity, decreased dexterity, and extreme tremor (Louis, 2009).

Patients with PD are typically suffering from non-motor symptoms. Cognitive impairment (CI) is the most debilitating non-motor PD symptom. In conjunction with both motor signs and dementia, the disorder puts a major social and economic strain on both caregivers and society. As a result, there were requests to determine the cause and risk factors of CI in individuals with PD. (Svenningsson et al., 2012) particularly with no treatment available to delay or prevent CI in PD. The entire spectrum of cognitive disability can be found in PD, ranging from subjective cognitive impairment to mild cognitive impairment (MCI) and PD dementia (PDD) (Baschi et al., 2018). The mean prevalence of PD-MCI was reported to be $26.7 \%$ (Litvan et al., 2011), but varies from 20 to 69 percent depending on the recruitment source and cognitive batteries used (Yarnall et al., 2014; Domellof et al., 2015). The underlying brain pathology contributing to PD cognitive deficits is still poorly known. Interruption of the circuitry that regulates the striatopallidothalamic network to the dorsolateral prefrontalcortex due to striatal dopaminergic deficit has been suggested to underlie cognitive impairment in PD (Emre, 2003).

Current research focus on the identification of early signs of diseases so that future medical assistance can postpone their growth (Pereira et al., 2019). The Self-Administered Gerocognitive Test (SAGE) (Scharre et al., 2010) is a widely used tool for cognitive assessment of MCI and early symptoms of dementia. SAGE is commonly used to classify signs and development of MCI, AD, and PD (Athilingam et al., 2015). Recently, a variety of works have focused on providing automated cognitive and motor function self-assessment tests on electronic consumer devices, such as smartphones (Aghanavesi et al., 2017), laptops (Sisti et al., 2017) and dedicated graphics tablets (Sole-Casals et al., 2019). Commonly conducted studies involve the tasks of Archimedes spiral drawing (Aghanavesi et al., 2017; Chen et al., 2018; Lopez-de-Ipina et al., 2018), finger tapping (Sisti et al., 2017), freehand drawing tasks (Lin et al., 2018), and tracing tasks (Chen et al., 2018). Methods used to analyse the collected spatiotemporal finger tapping, finger tapping, or pen route data include statistical analysis (San Luciano et al., 2016), discrete cosine transform (DCT) features (Sole-Casals et al., 2019), entropy, and fractal dimension analysis (Lopez-de-Ipina et al., 2018). 


\section{$\underline{\text { Aims: }}$}

The current protocol focuses on the development of a computerized model/smart phone application that assesses the cognitive and motor deficits of PD patients at the early stage, thus helping in delaying the progression of disease symptoms and providing more years of healthy life. Dementia Coach App is the proposed name for this new app.

\section{Hypothesis:}

Our hypothesis is developing a mobile app with an easy friendly interface that is available for download freely in Apple store and Google play store will help PD patients and their families to manage early cognitive/motor signs and symptoms with better management. This will delay the development of more serious problems related events for PD patients. Also this app will enable PD patient to live normal daily life.

\section{Methods}

\section{Patients recruitments:}

Ethical approval, Sample calculation and Patient selection including exclusion and inclusions criteria will be done accordingly. Grading and full clinical assessment for PD patients will be done according to established clinical scoring protocols.

\section{Equipment}

All measures and methods are based on Brouillette et al., (2013) and Lauraitis et al., (2020). However with the help and the guidance of IT, we can develop our own matrix and tasks within the app.

\section{Expected Outcomes and relevance:}

Mobile devices can be used to provide and monitor clinical care in PD patients as well as to track the health status of PD patients. Smartphone-based technologies have been used, for example, to address a number of psychological disorders, such as regeneration, depression and regression in the nongeriatric population (Rogante et al., 2010; Dufau et al., 2011; Mellone et al., 2012).

Smartphones have been described as an effective means of involvement in any intervention and to provide an effective means of tracking implementation and assessment of results. It is also important to stress that the effects of regional, clinical, patient diversity and recruitment costs are likely to influence how people are recruited for clinical research (Dufau et al., 2011).

This will allow further studies, more detailed studies, and the use of smartphones in geriatric research to be performed across a wider range of research. Dementia Coach App will come with following advantages: 1) Most of people are already engaged in online communities; (2) Privacy is maintained, thus overcoming sociocultural stigma associated with mental health care in Malaysia; (3) eTherapy eliminates physical barriers to access, which is especially important for women, remote areas and sexual minorities. Needless to say, it is a must tool during the current Pandemic of C19 with restricted MCO policy in Malaysia. The eTherapy selected is ideal because it focuses on core underlying mechanisms of effective therapeutic change, including exposure and skills building.

\section{References}

1. Olshansky SJ, Goldman DP, Zheng Y, Rowe JW (2009) Aging in America in the twenty-first century: demographic forecasts from the MacArthur Foundation Research Network on an Aging Society. Milbank Q 87: 842-862. 
2. Levine SR, Gorman M (1999) "Telestroke": the application of telemedicine for stroke. Stroke 30: 464-469.

3. Rosser JC Jr, Gabriel N, Herman B, Murayama M (2001) Telementoring and teleproctoring. World J Surg 25: 1438-1448.

4. Rajan R (2012) The promise of wireless: an overview of a device-to-cloud mHealth solution. Biomed Instrum Technol Fall Suppl: 26-32.

5. Clifford GD, Clifton D (2012) Wireless technology in disease management and medicine. Annu Rev Med 63: 479-492.

6. Rogante M, Grigioni M, Cordella D, Giacomozzi C (2010) Ten years of telerehabilitation: A literature overview of technologies and clinical applications. NeuroRehabilitation 27: 287-304.

7. Panayides A, Pattichis MS, Pattichis CS, Schizas CN, Spanias A, et al. (2010) An overview of recent end-to-end wireless medical video telemedicine systems using 3G. Conf Proc IEEE Eng Med Biol Soc 2010: 1045-1048.

8. Koch S (2006) Home telehealth-current state and future trends. Int J Med Inform 75: 565-576.

9. Fortney JC, Burgess JF Jr, Bosworth HB, Booth BM, Kaboli PJ (2011) A re- conceptualization of access for 21st century healthcare. J Gen Intern Med 26 Suppl 2: 639-647.

10. Boulos MN, Wheeler S, Tavares C, Jones R (2011) How smartphones are changing the face of mobile and participatory healthcare: an overview, with example from eCAALYX. Biomed Eng Online 10: 24.

11. Doherty ST, Oh P (2012) A multi-sensor monitoring system of human physiology and daily activities. Telemed J E Health 18: 185-192.

12. Dufau S, Dunabeitia JA, Moret-Tatay C, McGonigal A, Peeters D, et al. (2011) Smart phone, smart science: how the use of smartphones can revolutionize research in cognitive science. PLoS One 6: e24974.

13. Gill PS, Kamath A, Gill TS (2012) Distraction: an assessment of smartphone usage in health care work settings. Risk Manag Healthc Policy 5: 105-114.

14. Martin CK, Correa JB, Han H, Allen HR, Rood JC, et al. (2012) Validity of the remote food photography method (RFPM) for estimating energy and nutrient intake in near real-time. Obesity 20: 891-899.

15. Mellone S, Tacconi C, Schwickert L, Klenk J, Becker C, et al. (2012) Smartphone-based solutions for fall detection and prevention: the FARSEEING approach. Z Gerontol Geriatr 45: $722-727$.

16. Mellone S, Tacconi C, Chiari L (2012) Validity of a smartphone-based instrumented timed up and go. Gait Posture 36: 163-165.

17. Klasnja P, Pratt W (2012) Healthcare in the pocket: mapping the space of mobile-phone health interventions. J Biomed Inform 45: 184-198.

18. Worringham C, Rojek A, Stewart I (2011) Development and feasibility of a smartphone, ECG and GPS based system for remotely monitoring exercise in cardiac rehabilitation. PLoS One 6: e 14669.

19. Mack, J.; Marsh, L. Parkinson's Disease: Cognitive Impairment. Focus 2017, 15, 42-54.

20. Ludlow, C.L.; Connor, N.P.; Bassich, C.J. Speech timing in Parkinson's and Huntington's disease. Brain Lang.1987, 32, 195-214.

21. Marder, K.; Zhao, H.; Eberly, S.; Tanner, C.M.; Oakes, D.; Shoulson, I.; Huntington Study Group. Dietary intake in adults at risk for Huntington disease: Analysis of PHAROS research participants. Neurology 2009, 73, 385-392.

22. Louis, E.D. Essential tremors: A family of neurodegenerative disorders? Arch. Neurol. 2009, $66,1202-1208$.

23. Pereira, C.R.; Pereira, D.R.; Weber, S.A.T.; Hook, C.; de Albuquerque, V.H.C.; Papa, J.P. A survey on computer-assisted Parkinson's Disease diagnosis. Artif. Intell. Med. 2019, 95, 48-63.

24. Scharre, D.W.; Chang, S.-I.; Murden, R.A.; Lamb, J.; Beversdorf, D.Q.; Kataki, M.; Nagaraja, H.N.; Bornstein, R.A. Self-administered gerocognitive examination (SAGE). Alzheimer Dis. Assoc. Disord. 2010, 4, 64-71.

25. Athilingam, P.; Visovsky, C.; Elliott, A.F.; Rogal, P.J. Cognitive Screening in Persons with Chronic Diseases in Primary Care. Am. J. Alzheimer Dis. Other Dement. 2015, 30, 547-558.

26. Aghanavesi, S.; Nyholm, D.; Senek, M.; Bergquist, F.; Memedi, M. A smartphone-based 
system to quantify dexterity in parkinson's disease patients. Inform. Med. Unlocked 2017, 9, $11-$ 17.

27. Sisti, J.A.; Christophe, B.; Seville, A.R.; Garton, A.L.A.; Gupta, V.P.; Bandin, A.J.; Pullman, S.L. Computerized spiral analysis using the iPad. J. Neurosci. Methods 2017, 275, 50-54.

28. Solé-Casals, J.; Anchustegui-Echearte, I.; Marti-Puig, P.; Calvo, P.M.; Bergareche, A.; Sánchez-Méndez, J.I.; Lopez-de-Ipina, K. Discrete cosine transform for the analysis of essential tremor. Front. Physiol. 2019, 9, 1947.

29. Aghanavesi, S.; Memedi, M.; Dougherty, M.; Nyholm, D.; Westin, J. Verification of a method for measuring Parkinson's disease related temporal irregularity in spiral drawings. Sensors 2017, 17, 2341.

30. Chen, K.; Lin, P.; Yang, B.; Chen, Y. The di_erence in visuomotor feedback velocity control during spiral drawing between Parkinson's disease and essential tremor. Neurol. Sci. 2018, 39, $1057-1063$.

31. Lopez-de-Ipina, K.; Solé-Casals, J.; Faúndez-Zanuy, M.; Calvo, P.M.; Sesa, E.; Roure, J.; Bergareche, A. Automatic analysis of archimedes0 spiral for characterization of genetic essential tremor based on shannon's entropy and fractal dimension. Entropy 2018, 20, 531.

32. Sadikov, A.; Groznik, V.; Možina, M.; Žabkar, J.; Nyholm, D.; Memedi, M.; Georgiev, D. Feasibility of spirography features for objective assessment of motor function in parkinson's disease. Artif. Intell. Med. 2017, 81, 54-62.

33. San Luciano, M.; Wang, C.; Ortega, R.A.; Yu, Q.; Boschung, S.; Soto-Valencia, J.; SaundersPullman, R. Digitized spiral drawing: A possible biomarker for early parkinson's disease. PLoS ONE 2016, 11, e0162799.

34. Zham, P.; Arjunan, S.P.; Raghav, S.; Kumar, D.K. E_cacy of guided spiral drawing in the classification of parkinson's disease. IEEE J. Biomed. Health Inform. 2018, 22, 1648-1652.

35. Lin, P.; Chen, K.; Yang, B.; Chen, Y. A digital assessment system for evaluating kinetic tremor in essential tremor and parkinson's disease. BMC Neurol. 2018, 18, 25.

36. John V. Hindle. Ageing, neurodegeneration and Parkinson's disease. Age and Ageing, 2010 March;39(2):156-161.

37. Diane B. Miller and James P. O'Callaghan.Biomarkers of Parkinson's disease: Present and future.Metabolism. 2015 Mar; 64(3 0 1): S40-S46.

38. Svenningsson $P$, Westman E, Ballard C, Aarsland D. Cognitive impairment in patients with Parkinson's disease: diagnosis, biomarkers, and treatment. Lancet Neurol. (2012) 11:697-707. 10.1016/S1474-4422(12)70152-7

39. Baschi R, Nicoletti A, Restivo V, Recca D, Zappia M, Monastero R(2018) Frequency and correlates of subjective memory complaintsin Parkinson's disease with and without mild cognitive impairment:data from the Parkinson's disease cognitive impairment study. JAlzheimer's dis. J Alzheimers Dis 63(3):1015-1024

40. Litvan I, Aarsland D, Adler CH et al (2011) MDS task force on mildcognitive impairment in Parkinson'sdisease:criticalreviewofPD-MCI. Mov Disord 15(10):18141824

41. Yarnall AJ, Breen DP, Duncan GW, ICICLE-PD Study Group(2014) Characterizing mild cognitive impairment in incidentParkinson disease: the ICICLE-PD study. Neurology 82(4):308-3167.

42. Domellöf ME, Ekman U, Forsgren L, Elgh E (2015) Cognitivefunction in the early phase of Parkinson's disease, a five-year fol-low-up. Acta Neurol Scand 132(2):79-88

43. Emre M (2003) What causes mental dysfunction in Parkinson'sdisease? Mov Disord 8(Suppl 6):S63-S71 